OPEN ACCESS

Edited by:

Daniela Ceccarelli,

Central Veterinary Institute Wageningen University and Research

Centre, Netherlands

Reviewed by:

Nicolas Carraro,

Université de Sherbrooke, Canada

Genevieve Garriss,

Karolinska Institutet, Sweden

*Correspondence:

Lisbeth E. de Vries

lidv@phmetropol.dk

Specialty section:

This article was submitted to

Antimicrobials, Resistance

and Chemotherapy,

a section of the journal

Frontiers in Microbiology

Received: 27 November 2015

Accepted: 08 April 2016

Published: 26 April 2016

Citation:

de Vries LE, Hasman H, Jurado

Rabadán S and Agersø Y (2016)

Sequence-Based Characterization of

Tn5801-Like Genomic Islands

in Tetracycline-Resistant

Staphylococcus pseudintermedius and Other Gram-positive Bacteria

from Humans and Animals.

Front. Microbiol. 7:576.

doi: 10.3389/fmicb.2016.00576

\section{Sequence-Based Characterization of Tn5801-Like Genomic Islands in Tetracycline-Resistant Staphylococcus pseudintermedius and Other Gram-positive Bacteria from Humans and Animals}

\author{
Lisbeth E. de Vries ${ }^{1 *}$, Henrik Hasman², Sonia Jurado Rabadán ${ }^{3}$ and Yvonne Agersø² \\ ${ }^{1}$ Department of Technology, Metropolitan University College, Copenhagen, Denmark, ${ }^{2}$ National Food Institute, Technical \\ University of Copenhagen, Lyngby, Denmark, ${ }^{3}$ Animal Health Department, Universidad Complutense de Madrid, Madrid, \\ Spain
}

Antibiotic resistance in pathogens is often associated with mobile genetic elements, such as genomic islands (GI) including integrative and conjugative elements (ICEs). These can transfer resistance genes within and between bacteria from humans and/or animals. The aim of this study was to investigate whether Tn5801-like Gls carrying the tetracycline resistance gene, tet(M), are common in Staphylococcus pseudintermedius from pets, and to do an overall sequences-based characterization of Tn5801-like Gls detected in Gram-positive bacteria from humans and animals. A total of 27 tetracycline-resistant S. pseudintermedius isolates from Danish pets (1998-2005) were screened for tet(M) by PCR. Selected isolates (13) were screened for Gl- or ICE-specific genes (int $T_{\text {Tn581 }}$ or $x_{\text {is }}{ }_{\text {Tn916) }}$ ) and their tet(M) gene was sequenced (Sanger-method). Long-range PCR mappings and whole-genome-sequencing (Illumina) were performed for selected S. pseudintermedius-isolates (seven and three isolates, respectively) as well as for human S. aureus isolates (seven and one isolates, respectively) and one porcine Enterococcus faecium isolate known to carry Tn5801-like Gls. All 27 S. pseudintermedius were positive for tet(M). Out of 13 selected isolates, seven contained Tn5801-like Gls and six contained Tn916-like ICEs. Two different Tn5801like Gl types were detected among S. pseudintermedius (Tn5801 and Gl6287) - both showed high similarity compared to GenBank sequences from human pathogens. Two distinct Tn5801-like Gl types were detected among the porcine E. faecium and human S. aureus isolates (Tn6014 and Gl6288). Tn5801-like Gls were detected in GenBanksequences from Gram-positive bacteria of human, animal or food origin worldwide. Known Tn5801-like Gls were divided into seven types. The results showed that Tn5801like Gls appear to be relatively common in tetracycline-resistant S. pseudintermedius in Denmark. Almost identical Tn5801-like Gls were identified in different Gram-positive species of pet and human origin, suggesting that horizontal transfer of these elements has occurred between S. pseudintermedius from pets and human pathogens, including S. aureus.

Keywords: tet(M), integrative and conjugative elements, ICEs, GIs, transmission, horizontal gene transfer, Tn916, mobile genetic elements 


\section{INTRODUCTION}

The emergence of antibiotic resistance among pathogenic bacteria is a general problem which is regarded as a threat to public health in many countries - both human and veterinary infections caused by antibiotic resistant pathogens pose a challenge to treatment and have high economic costs (Marshall and Levy, 2011; Wernli et al., 2011; Wegener, 2012; U.S. Department of Health and Human Services - Centers for Disease Control and Prevention [CDC], 2013) ${ }^{1}$.

Resistant bacteria can develop in a human or animal host by evolutionary selection due to the presence of antibiotics, e.g., in connection with antibiotic therapy (Davies and Davies, 2010; Marshall and Levy, 2011). In addition, a host can acquire resistant bacteria by transmission from an outside source, e.g., via contact with other people and/or animals or via environmental exposure (Guardabassi et al., 2004a; Wegener, 2012; Da Costa et al., 2013). Transmission of multiple antibiotic resistant Staphylococcus pseudintermedius between dogs and humans has been reported (Guardabassi et al., 2004a). S. pseudintermedius is the most important staphylococcal pathogen in dogs where it is primarily associated with skin and ear infections, but it rarely causes infections in humans. Occasionally, this species is isolated from skin infection in cats (Bannoehr and Guardabassi, 2012).

Antibiotic resistance in pathogens is often associated with mobile genetic elements (MGEs) that can transfer antibiotic resistance genes within and between bacteria from human and/or animal hosts (Burrus et al., 2002; Bennett, 2008; Roberts and Mullany, 2011). MGEs include plasmids, transposable elements, prophages and different types of genomic islands (GIs) such as integrative and conjugative elements (ICEs) (Wozniak and Waldor, 2010).

Tn5801-like GIs belong to the family of Tn916-like ICEs. Tn916 was originally identified in the late 1970's as an 18$\mathrm{kb}$ conjugative transposon from Enterococcus faecalis DS16 (Franke and Clewell, 1981). Many Tn916-like ICEs have a broad host range and are responsible for dissemination of the tetracycline resistance gene tet $(\mathrm{M})$ in Gram-positive bacteria from humans and animals (Franke and Clewell, 1981; Rice, 1998; Roberts and Mullany, 2011). Tn5801 is a putative ICE (25.3 kb), however, this has not been proven. Like Tn916, Tn5801 is also associated with the tet $(\mathrm{M})$ gene. The tet $(\mathrm{M})$ gene encodes a ribosomal protection protein conferring resistance by protecting the ribosome against the action of tetracyclines (Chopra and Roberts, 2001). Tn5801 was first described in a clinical S. aureus isolate, Mu50, from a Japanese boy (Kuroda et al., 2001). Tn5801-like GIs have subsequently been identified in other human S. aureus and Streptococcus isolates, mainly in pathogenic, but also in commensal strains (Kuroda et al., 2001; de Vries et al., 2009; Denapaite et al., 2010; Holden et al., 2010; Mingoia et al., 2013; Brenciani et al., 2014). Comparative genome hybridization analysis recently showed Tn5801 to be specifically associated with human methicillin-resistant $S$. aureus (MRSA) in China (Li et al., 2013). Only a few Tn5801-like

${ }^{1}$ Centers for Disease Control and prevention (CDC): http://.cdc.gov/features/anti bioticresistance/ elements from animal-associated bacterial isolates have been reported so far: a partially sequenced element, CW459tet(M) in Clostridium perfringens isolated from porcine feces, and an element detected in an E. faecium isolate CICYT-205 from a healthy pig in Spain (Roberts et al., 2001; Jurado-Rabadán et al., 2014). For the latter, only tet(M) was sequenced. Finally, a Tn5801-like GI has recently been reported in a fully sequenced genome from a methicillin resistant S. pseudintermedius (MRSP) strain ED99 isolated from a dog in the UK (Ben Zakour et al., 2012).

The importance of GIs/ICEs associated with tet $(\mathrm{M})$ was highlighted in a recent study. Previous extensive clinical use of tetracycline and corresponding integration of Tn916- and Tn5801-like elements in Streptococcus agalactiae was shown to have had great significance for the dissemination of specific pathogenic S. agalactiae clones in humans (Da Cunha et al., 2014).

Characteristic of MGEs, including the Tn916-like family, Tn5801-like elements have a modular organization (Figure 1A; Burrus et al., 2002; Toussaint and Merlin, 2002; Roberts and Mullany, 2009). Tn5801 contains ORFs similar to those of Tn916, but differs by containing an integrase gene $\left(\right.$ int $\left._{\mathrm{Tn} 5801}\right)$ different from the excisionase/integrase genes $\left(x_{i s_{\mathrm{Tn} 916}} /\right.$ int $\left._{\mathrm{Tn} 916}\right)$ present in Tn916. The int $_{\mathrm{Tn} 5801^{-}}$and int $_{\mathrm{Tn} 916}$ genes are of different sizes and show very low DNA identity $(<50 \%)$ - they encode different types of tyrosine recombinases. The int $t_{\mathrm{Tn} 5801}$ gene is identical to the int459 gene from the element CW459tet(M) (Roberts et al., 2001). Both Tn5801 and CW459tet(M) are integrated at the same site, located at the $3^{\prime}$ end of an ORF, predicted to encode GMP synthetase, whereas Tn916 can integrate at random sites in most hosts, but preferentially into AT-rich sequences (Burrus et al., 2002). The tet(M) genes from Tn5801 and Tn916 show high similarity (97.7\% DNA identity). Only

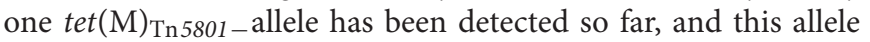
appears to be specifically associated with Tn5801-like GIs (de Vries et al., 2009).

The overall aim of this study was to investigate whether Tn5801-like elements carrying tet(M) are common in clinical S. pseudintermedius isolates from pets, where they may constitute a likely reservoir for human pathogens (Guardabassi et al., $2004 a, b)$. Another aim of the study was to perform an overall sequence-based characterization of Tn5801-like GIs detected in Gram-positive bacteria from humans and animals in order to get an overview of different Tn5801-like GI types and the degree of Tn5801-like GI dissemination. The study was performed by screening selected tetracycline-resistant $S$. pseudintermedius from veterinary diagnostic isolates for tet $(\mathrm{M})$ as well as for int or xis genes specific for Tn5801- or Tn916-like elements, respectively. PCR mapping and gene/genome sequencing was performed in order to characterize and compare different Tn5801-like GIs detected in pet-associated S. pseudintermedius isolates as well as in human $S$. aureus isolates and one pig E. faecium isolate from previous studies (de Vries et al., 2009; Jurado-Rabadán et al., 2014). We conducted an overall comparison of full length Tn5801-like GIs characterized in this study and detected in genome sequences submitted to GenBank. 


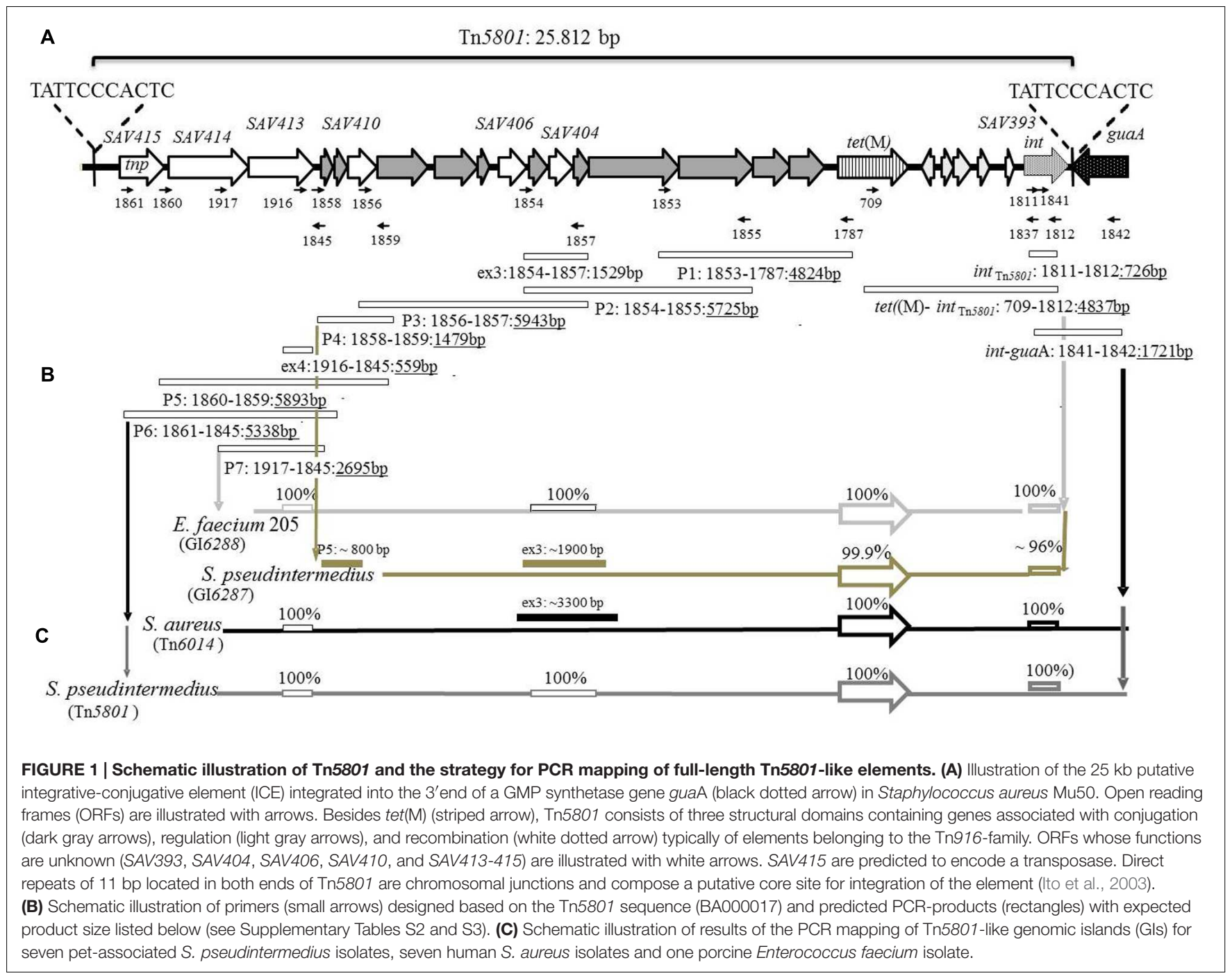

\section{MATERIALS AND METHODS}

\section{Strains}

Twenty-seven tetracycline-resistant isolates from dogs and one cat were sampled and identified as $S$. intermedius by biochemical tests, morphology and 16S sequencing (Supplementary Table S1). Based on host origin and PCR-screening, all isolates were further identified as S. pseudintermedius (Bannoehr and Guardabassi, 2012). All isolates were diagnostic submissions to The National Veterinary Institute, Technical University of Denmark. Thirteen S. pseudintermedius isolates selected to cover the whole isolation period, 1998-2005 were characterized further in regard to tet(M) and Tn5801-like sequences (Table 1). In addition, one porcine E. faecium isolate and seven human $S$. aureus isolates identified in previous studies were also characterized (Table 1). All $13 \mathrm{~S}$. pseudintermedius isolates, except one isolate, 200007910-1, were confirmed to be tetracycline-resistant by the use of Sensititre and MIC testing (Aarestrup et al., 2000a). Susceptibility MIC-testing using cefoxitin showed all 13 isolates to be methicillin sensitive S. pseudintermedius (MSSP).
Besides tetracycline and cefoxitin, the isolates were also tested for susceptibility to penicillin, streptomycin, erythromycin, ciprofloxacin, spectinomycin, tiamulin, trimethoprim, chloramphenicol, florfenicol, gentamicin, and sulfamethoxazole by the Sensititre method as described previously (Aarestrup et al., 2000a).

\section{PCR Screening and Sequencing of tet(M), int ${ }_{\mathrm{Tn} 5801}$ and $\mathrm{xis}_{\mathrm{Tn} 916}$ in S. pseudintermedius}

All 27 S. pseudintermedius isolates were screened for tet(M) by PCR as described previously (see Supplementary Table S2; Aarestrup et al., 2000b). Thirteen tet(M)-positive isolates (Table 1) were selected for tet(M)-gene-sequencing by the Sanger method in a manner similar to a previously described strategy (see Supplementary Information; de Vries et al., 2009). In addition, the 13 isolates were screened for int $t_{\mathrm{Tn} 5801}$ or $x$ s $_{\text {Tn } 916}$ genes by PCR, and selected screening products were sequenced by the Sanger method and compared to sequences 


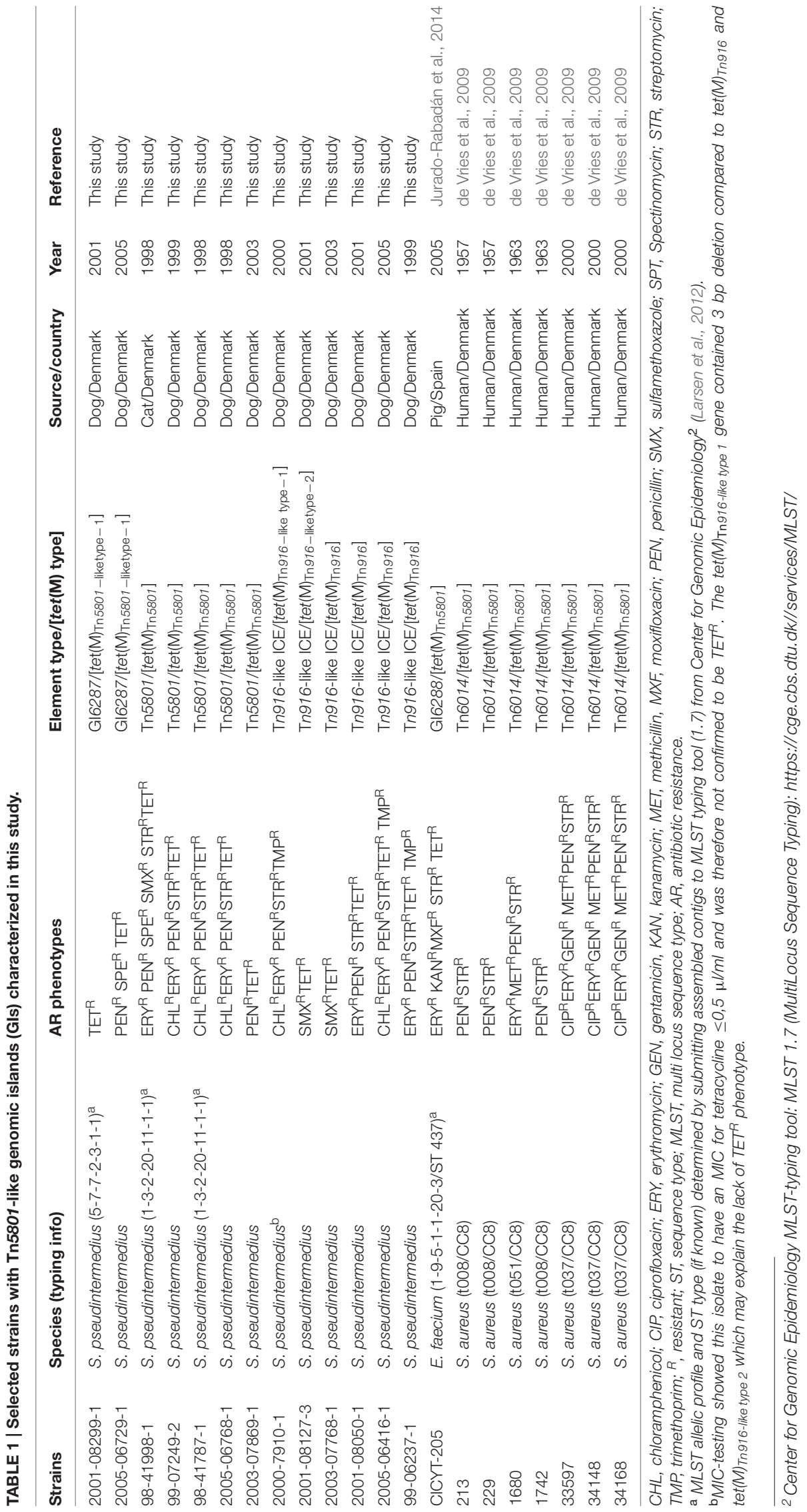


from Tn5801 and Tn916 as described in former studies (Agerso et al., 2002; de Vries et al., 2009). (See Supplementary Information, including Supplementary Tables S2 and S3 for further details).

\section{PCR Mapping of Tn5801-Like Gls from Pet-Associated S. pseudintermedius, Human S. aureus and a Porcine E. faecium}

The strategy for mapping the Tn5801-like elements is outlined in Figures 1A,B. Elements detected in pet-associated S. pseudintermedius from this study and previously detected elements in isolates from human $S$. aureus and porcine E. faecium were mapped (de Vries et al., 2009; Jurado-Rabadán et al., 2014). The mapping was performed by Long range PCR with the Phusion ${ }^{\text {TM }}$ High-Fidelity DNA Polymerase (Finnzymes, Finland) using the conditions recommended by the manufacturer. DNA Taq polymerase (Ampliqon, Denmark) was used to obtain PCR products expected to be $<2000 \mathrm{bp}$. The PCR conditions and primers are listed in Supplementary Tables S2 and S3. S. aureus Mu50 was used as positive control and a $S$. pseudintermedius strain 200307768-1 that was shown to be negative for int $t_{\mathrm{Tn} 5801}$ and positive for $x i_{\mathrm{Tn} 916}$, was used as negative control. Besides int ${ }_{\mathrm{Tn} 5801}$, selected PCRproducts, ex3 and ex4, were fully or partially sequenced by the Sanger method and compared to corresponding sequences in Tn5801.

\section{Whole-Genome Sequencing of Selected S. pseudintermedius, S. aureus, and E. faecium}

Based on the PCR mapping of detected Tn5801-like elements and tet $(\mathrm{M})$ sequence types $3 \mathrm{~S}$. pseudintermedius strains (2001-08299-1, 9841998-1, and 9841787-1), S. aureus 1680 and E. faecium CICYT-205 were selected for whole-genome sequencing. Genomic DNA was purified by using the Easy-DNA gDNA Purification kit (Life Sciences). For each isolate, a sequencing library was constructed and pooled using Nextera DNA Sample Preparation Kit (Illumina). Sequencing was performed using the Illumina platform, MiSeq (paired-end reads). Paired-end reads were trimmed directly by the MiSeq in order to remove sequencing adaptor and primer contamination, and CLC Genomic Workbench (version 7.5.1) was used to remove low quality reads. De novo assembly was conducted using CLC Genomic Workbench (version 7.5.1). (See Supplementary Information including Supplementary Tables S4 and S5 for details regarding trimming and assembly). For all five isolates, assembled contigs of minimum 500 bp (for CICYT-205 cut-off was minimum 1000 bp contigs) with an average coverage $>30 \mathrm{x}$ were submitted as a Whole Genome Shutgun (WGS) sequencing project to GenBank under bioProject: PRJNA264198; bioSamples: SAMN03120277, SAMN03120291, SAMN03120292, SAMN03120293, SAMN03120290; accession no: JTKN00000000, JTKO00000000, JTKP00000000, JTKQ00000000, JTKR00000000. The WGS submissions were annotated by NCBI Prokaryotic
Genome Automatic Annotation Pipeline (PGAAP) ${ }^{3}$ (Angiuoli et al., 2008).

\section{Bioinformatic Analysis of Sequenced Genomes with Focus on Tn5801-Like Gls}

For all the five WGS-sequences, species were confirmed by using the strain identification tool (EzTaxon server) ${ }^{4}$ and/or the species finder tool (SpeciesFinder 1.0) ${ }^{5}$ using annotated full length $16 \mathrm{~S}$ rRNA sequences and/or assembled contigs as input, respectively (Larsen et al., 2014). Besides, all five WGS-sequences were analyzed for MLST allelic profile, ST type and the presence of antibiotic resistance genes using the online-tools MLST typing tool (1.7) and ResFinder ${ }^{6}$ (Table 1 and Supplementary Table S6) (Zankari et al., 2012). Contigs containing Tn5801-like tet(M) and int $\operatorname{Tn} 5801$ sequences from the sequenced genomes were detected by search annotations for "tet(M)" and "int" as well as sequences for identity to int $t_{\operatorname{Tn} 5801}$. For all staphylococci isolates a Tn5801like element was detected in one contig, whereas for the E. faecium CICYT-205 isolate, specific Tn5801-like sequences were detected in two contigs that showed an overlap of 129 bp with 100\% DNA identities. The two contigs were fused in Geneious (Trial version 8.0.4). Primers used in the PCR mapping were mapped to the selected contigs containing Tn5801-like elements, and expected PCR-product sizes were compared to product sizes obtained in the PCR mapping in order to verify correct assembly. Sequence analysis was performed using CLC Genomic Workbench (version 7.5.1 or 8.5.1). GenBank was searched for full length Tn5801like GIs based on a BLASTp ${ }^{7}$ search using the annotated Int protein of Tn5801 as query (GenBank accession no. BAB56554). A threshold of minimum 98\% amino acid (aa.) identity was used, and 11 full-length elements for which sequences were published were selected for comparative sequence analysis together with elements sequenced in this study.

\section{RESULTS}

\section{The tet(M) Gene in S. pseudintermedius from Pets Was Detected on Tn5801- and Tn916-Like Elements}

Twenty seven tetracycline-resistant clinical S. pseudintermedius isolates were screened for the presence of the tet $(\mathrm{M})$ gene. All 27 isolates were positive for the tet(M)-PCR screening (Supplementary Table S1). Of the 13 sequenced tet $(\mathrm{M})$ genes, seven were highly similar to tet $(\mathrm{M})_{\mathrm{Tn} 5801}$ from S. aureus Mu50 (99.9-100\% DNA identity), and six sequences were highly similar

\footnotetext{
${ }^{3}$ PGAAP: http://www.ncbi.nlm.nih.gov/genome/annotation_prok

${ }^{4}$ E. Strain identification tool: http://www.ezbiocloud.net/eztaxon

${ }^{5}$ Center for Genomic Epidemiology SpeciesFinder 1.0 Server: https://cge.cbs.dtu. $\mathrm{dk} /$ services/SpeciesFinder/

${ }^{6}$ Center for Genomic Epidemiology Antibiotic resistance finder tool: https://cge.cb s.dtu.dk//services/ResFinder/

${ }^{7}$ NCBI BLAST. http://blast.ncbi.nlm.nih.gov/Blast.cgi
} 
to tet $(\mathrm{M})_{\operatorname{Tn} 916}$ from $E$. faecalis DS16 (99.8-100\% DNA identity). Altogether two Tn5801-like tet(M)-sequence types were detected: tet $(\mathrm{M})_{\text {Tn } 5801}$ and tet $(\mathrm{M})_{\text {Tn } 5801-l i k e-t y p e-1}$ differing by 1/1920 bp. In addition, 3 Tn916-like-tet $(\mathrm{M})$ sequence types were detected:

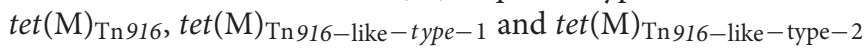
differing by $1-3 / 1920$ bp. (See Table 1 and Supplementary Table S7 for details). PCR screenings confirmed that the 13 tested strains contained int $_{\operatorname{Tn} 5801}$ or $x i s_{\operatorname{Tn} 916}$ genes. In addition, long PCR confirmed that the 7 Tn5801-like tet(M) genes were physically linked to detected int $t_{\mathrm{Tn} 5801}$ genes (see Figures 1B,C).

\section{Two Different Tn5801-Like Gls Types Were Detected Among the S. pseudintermedius}

PCR mapping and whole genome sequencing revealed two different Tn5801-like elements among the tested S. pseudintermedius isolates corresponding to the two different Tn5801-like tet(M) types detected (see Figures 1 and 2A).

For 5 S. pseudintermedius isolates, the PCR mapping showed that all amplified PCR products were of the same size compared to the control strain Mu50 (Figures 1B,C and Table 1). Besides tet $(\mathrm{M})_{\mathrm{Tn} 5801}$, the int $_{\mathrm{Tn} 5801}$ screening product and sequenced ex4- and ex3 fragments from these strains were shown to be $100 \%$ identical to corresponding regions in Tn5801 (Figures 1B,C). The whole element present in theses isolates was shown to be almost identical to Tn5801 from S. aureus Mu50 both in organization as well as on DNA sequence level (Table 2). In addition, all ORFs of this element were identical or highly similar to Tn5801 ORFs (int Tn5801-sav415; $_{\text {; }}$ Figure 2A).

For two other $S$. pseudintermedius strains, the PCR mappings were positive, except for int-guaA, ex4, P6 and P7. The size of P2, P3, ex3, and P5 differed from Tn5801 (Figures 1B,C). These

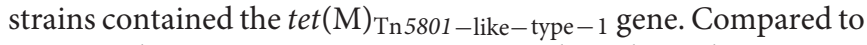
Tn5801, the int $_{\mathrm{Tn} 5801-l i k e}$ screening product showed $96 \%$ DNA identity. This element showed similar organization compared to Tn5801, but differed by being approximately 5000 bp smaller than Tn5801 corresponding to a missing upstream region containing sav413-sav415 of Tn5801 (Figure 2A and Table 2). Instead of Tn5801 sav404, this element contained a larger ORF with low similarity to sav404. Thus, this element was registered as a novel element, GI6287 in the Transposon Nomenclature Database from the UCL Eastman Dental Institute, London ${ }^{8}$ (Roberts et al., 2008; Roberts and Mullany, 2011).

Both S. pseudintermedius Tn5801 and GI6287 were found to be located in the $3^{\prime}$ end of putative GMP synthase genes (guaA) with high similarity (99.68\% DNA identity) and downstream of a putative integrase pseudogene with high similarity (97.45\% DNA identity). Both elements are flanked by 11 bp sequences that are almost identical to the direct repeats that form the core of the putative attachment sites of Tn5801 (Ito et al., 2003; Figure 2A).

${ }^{8}$ http://www.ucl.ac.uk/eastman/tn/

\section{Two Other Tn5801-Like GI Types Were Characterized from the Human S. aureus Isolates and One $E$. faecium Isolate from Pig}

Characterization of Tn5801-like GIs previously detected in human clinical S. aureus from Denmark and an E. faecium strain isolated from a healthy pig in Spain revealed 2 other Tn5801like GI types different from the elements detected among the S. pseudintermedius isolates (see Figures 1 and 2; de Vries et al., 2009; Jurado-Rabadán et al., 2014).

The PCR mapping showed that for the seven S. aureus isolates, PCR fragment P1, P4, ex4, P5-P7 were of the same size as Tn5801 (Figures 1B,C). PCR products P2, P3, and ex3 were approximately $1.7-\mathrm{kb}$ longer than the corresponding fragments detected in $\mathrm{Mu} 50$. As for $\operatorname{tet}(\mathrm{M})_{\mathrm{Tn} 5801}$, the int $\mathrm{Tn}_{\mathrm{Tn} 5801}$ screening product and the ex4 fragment showed 100\% DNA identity with Tn5801. This element is referred to as Tn6014 as previously registered (de Vries et al., 2009). The whole Tn6014 sequence contained identical or highly similar ORFs and showed overall similarity compared to Tn5801 ( int $_{\mathrm{Tn} 5801}$-sav415; Figure 2B and Table 2). In addition, Tn6014 contained an additional region of 1775 bp corresponding to two putative ORFs not present in Tn5801.

For the E. faecium isolate, all PCRs except for P6, P5 and int-guaA were shown to be of the same size as for Tn5801 (Figures 1B,C). Besides the tet $(\mathrm{M})_{\mathrm{Tn} 5801}$ gene sequenced previously (Jurado-Rabadán et al., 2014), the int Tn5801 $_{\text {screening }}$ product and the ex3 fragment showed 100\% DNA identity with Tn5801. This element showed similarity on DNA level and a similar organization compared to Tn5801, except that it did not contain a sav415-like ORF that is predicted to encode a transposase in Tn5801 (Figure 2C and Table 2). This element was registered as a novel element: GI6288.

Both Tn6014 and GI6288 were found integrated into the $3^{\prime}$ end of $g u a A$. The 11-bp sequences that correspond to the imperfect direct repeats included in the attL and attR sites that flank the elements were almost identical to those of Tn5801 (see Figures 2B,C).

\section{Sequence Analysis of S. aureus Tn6014}

Further sequence analysis (BLASTn search in GenBank) ${ }^{9}$ of Tn6014 from the human S. aureus strain showed that most of the additional 1775 bp-region detected in Tn6014 was highly similar (99.89\% DNA identity) to a region containing ORFs predicted to encode an integrase and a transposase in the genome of an E. faecalis strain 62 isolated from a stool sample of a healthy Norwegian infant (Brede et al., 2011). This isolate was reported to contain a Tn916 element at a different location (accession no. CP002491; 562138-582758) than the 1771 bp region similar to a region in Tn6014. However, we showed it to be a Tn5801like element containing int ${ }_{\mathrm{Tn} 5801}$ integrated into a guaA gene (see next paragraph). Thus, Tn6014 is shown to be a composite element that may have been formed within Gram-positives species, possibly Enterococcus.

\footnotetext{
${ }^{9}$ NCBI BLAST. http://blast.ncbi.nlm.nih.gov/Blast.cgi
} 


\section{A}

S. Pseudintermedius Tn5801-like GIs:

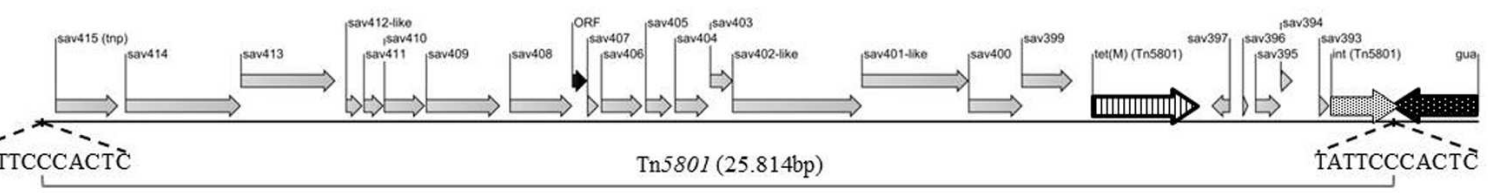

TATTCCCACT்̃ $\operatorname{Tn} 5801(25.814 \mathrm{bp})$

ṪÁTTCCCACTC̀

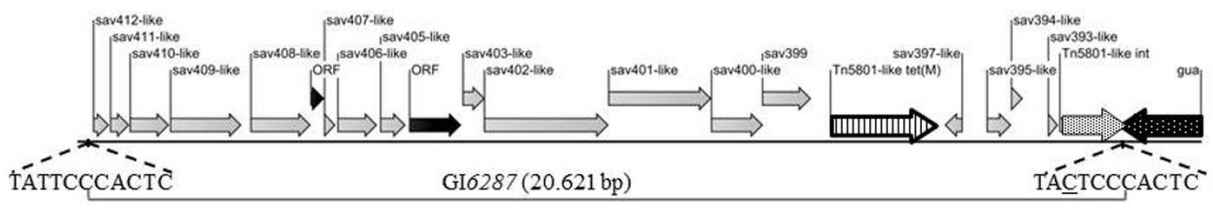

B

S. aureus Tn5801-like GI, Tn6014:

E. faecalis 62 region $(1771 \mathrm{bp})$ of genome (CP002491: 2189099-2190868)

EF62_2202: int core domain proteing ingly protein

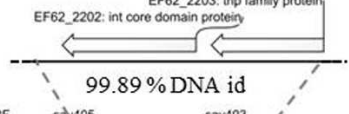

' $99.89 \%$ DNA id ,'

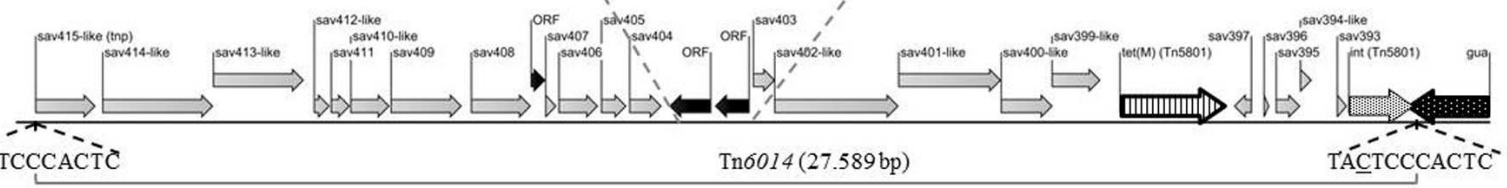

C

E. faecium Tn5801-like GI:

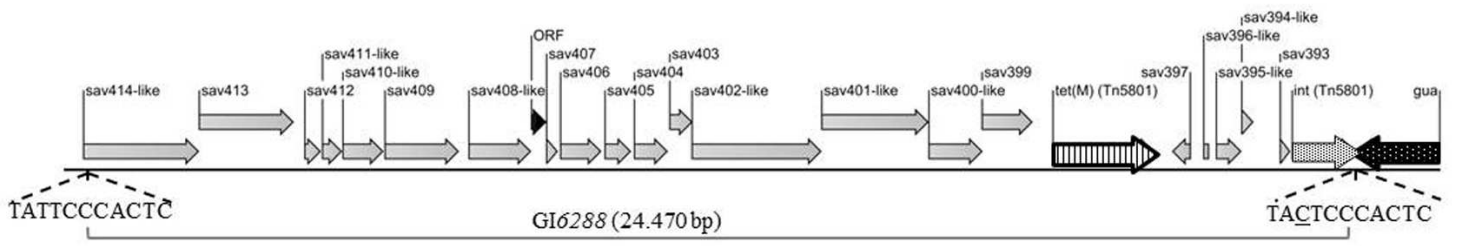

FIGURE 2 | Illustration of Tn5801-like Gls from Gram-positive bacteria fully sequenced in this study. Gray arrows illustrate ORFs identical (sav393-sav397, and sav399-sav415) or similar (sav393-like-sav415-like) to corresponding ORFs in Tn5801, black arrows illustrate ORFs which were not annotated in Tn5801. Striped arrow: tet(M), white dotted arrow: Tn5801-like integrase gene (int), black dotted arrow: guaA, tnp: tranposase. Direct repeats of 11 bp located in both ends of Tn5801-like elements are chromosomal junctions and compose a putative core site for integration of the element (Ito et al., 2003). Underlined nucleotides differ from Tn5801. (A) Tn5801 from S. pseudintermedius 9841787-1 isolated from a dog and 9841998-1 isolated from a cat - sequenced Gls from the two isolates were 100\% identical. Gl6287 from S. pseudintermedius 200108299-1 isolated from a dog. (B) Tn6014 from human S. aureus 1680 (C) Gl6288 sequenced from E. faecium CICYT205.

TABLE 2 | Comparison of whole Tn5801-like Gl-sequences from this study with corresponding sequences from Tn5801, S. aureus Mu50.

GI

$25807 / 25814$
$19172 / 25812$
$25782 / 27589$
$24451 / 25812$

DNA identity ${ }^{\mathrm{a}}(\%)$

99.97

73.12

93.45

94.72
S. pseudintermedius Tn5801
S. aureus Tn6014

$24451 / 25812$

Gaps (number of gaps ${ }^{a} /$ total $^{b} p^{b}$ )

aDetermined by pairwise alignment and comparison using CLC Genomic Workbench (version 8.5.1).

${ }^{\mathrm{b}}$ Corresponding to the largest of the compared Gls. 


\section{Comparison of Tn5801-Like Gls Shows Evidence of Horizontal Transfer between Gram-positive Species of Human and Animal Origin}

Tn5801-like GIs were found to be present in GenBank sequences from different Gram-positive bacterial pathogens and a few commensals, mainly from humans, but also sequences from bacteria associated with animals and food in Europe, USA, Asia, and Australia were found (see Supplementary Table S8). A multiple alignment of 11 selected full-length Tn5801-like GIs from GenBank and the five Tn5801-like GIs characterized in this study divided the elements into seven predicted GI types. All Tn5801-like GIs characterized in this study except for Tn6014 fell into three different groups (groups 1-3; Figure 3). Pairwise comparisons of fulllength elements within groups 1, 2, and 3 showed very high similarity. In addition to $S$. aureus Tn5801, S. pseudintermedius Tn5801 showed very high similarity to the Tn5801-like element from canine S. pseudintermedius ED99 within group 1 (99.98\%). Besides, S. pseudintermedius Tn5801 showed high similarity to an element from a Lactococcus garvieae IPLA31405 strain isolated from cheese (99.40\% DNA identities). Within group 2, E. faecium GI6288 showed 99.88\% DNA identity compared to an element from a human S. mitis B6 isolate. Within group 3, S. pseudintermedius GI6287 was shown only to differ by 4 out of 20,621 bp from corresponding elements in human isolates, E. faecalis strain 62 and S. agalactiae COOH1. This comparison shows that almost identical full-length Tn5801-like GIs are present in Gram-positive species of animal and human origin, which strongly supports the occurrence of recent direct or indirect horizontal transfer of these elements between different species of different origin. These results in particular support occurrence of horizontal transfer of Tn5801 and GI6287 between pet-associated S. pseudintermedius and Gram-positive pathogens of human origin.

\section{DISCUSSION}

Tetracycline resistance is common in $S$. pseudintermedius and is most often mediated by the tet(M) gene (Schwarz et al., 1998; Kim et al., 2005). In agreement with this, we found all the tetracycline-resistant $S$. pseudintermedius isolates in this study (27) to contain the tet(M) gene. We cannot completely rule out that tetracycline resistance could also partly be mediated by other genes, as this was not further investigated. However, this is unlikely, since no other tetracycline resistance genes were detected by the sequence analysis of the fully sequenced $S$. pseudintermedius genomes (Supplementary Table S6). For the thirteen isolates for which the tet(M) gene was sequenced, we showed that six and seven contained Tn916-like and Tn5801-like elements, respectively. This shows Tn5801-like GIs to be relatively common in tetracyclineresistant pet-associated S. pseudintermedius in Denmark, and the prevalence appears to be on the same level as for
Tn916-like ICEs. Previously, Tn5801-like GIs (Tn6014) were detected in 7 out of 20 tet(M)-positive human S. aureus isolates from Denmark (1957-2001; de Vries et al., 2009). As opposed to this, 17 porcine $S$. aureus strains from the same study only contained tet $(\mathrm{M})$ associated with Tn916like ICEs. A Tn5801-like element was recently reported in a MRSP canine strain (Schwarz et al., 1998; Ben Zakour et al., 2012). However, this study is the first to address the prevalence of Tn5801-like elements among tetracycline-resistant S. pseudintermedius and the first report on Tn5801-like elements within MSSP.

Previous studies have suggested that specific tet $(\mathrm{M})$-alleles or subtypes of tet $(\mathrm{M})$ are associated with a specific element type or element subgroups within the Tn916-like ICE family as well as other ICE families (Agerso et al., 2006; de Vries et al., 2009; Jurado-Rabadán et al., 2014). Among the tested S. pseudintermedius isolates with Tn5801-like GIs two different subtypes were detected corresponding with two highly similar tet $(\mathrm{M})$ sequence types that only differed by $1 / 1920 \mathrm{bp}$ corresponding to a difference of 1/640 aa. The two tet $(\mathrm{M})$ subtypes were shown both by Sanger and Illumina sequencing. This is the first report of a new Tn5801-like tet(M) subtype. This subtype may be specifically associated with GI6287, however, the almost identical Tn5801like GIs that are present in E. faecalis strain 62 and S. agalactiae COOH1 contain the known Tn5801-tet(M) provided that the difference does not result from a sequencing mistake.

Tn5801 was detected in the genome of S. pseudintermedius isolates from four dogs and a one cat, including two isolates of the same unknown sequence type (ST). S. pseudintermedius Tn5801 was shown to be almost identical compared to the element detected in the MRSP $S$. pseudintermedius ED99 (UK, ST type 25) and the Tn5801 element from the Japanese human $S$. aureus strain (Mu50). As mentioned above, S. pseudintermedius GI6287 was shown to be highly similar to elements present in human Streptococcus and Enterococcus strains from US and Norway, respectively. The fact that almost identical GIs can be found in different species of animal and human pathogens supports that horizontal transfer of these elements has occurred between the S. pseudintermedius and human pathogens - the direction of transfer cannot be determined, however. Alternatively, the different species may have received these elements from a common bacterial source.

The mechanism of transfer for Tn5801 is unknown, but the similar organization of Tn5801 and Tn916 may suggest that Tn5801, like Tn916, transfer horizontally by conjugation with a rolling circle (Rice, 1998; Mullany et al., 2002). The only evidence of horizontal transfer of an Tn5801-like element is presented in a previous study (de Vries et al., 2009). Tn6014 from human $S$. aureus strain 1680 was shown to transfer at very low frequencies to a recipient strain - actually only one transconjugant was shown to have received the Tn6014tet $(\mathrm{M})$ gene physically linked to the int $t_{\mathrm{Tn} 5801}$. Attempts to detect proof of circularization of Tn6014 by PCR did not succeed (data not shown). In this study we were not able 


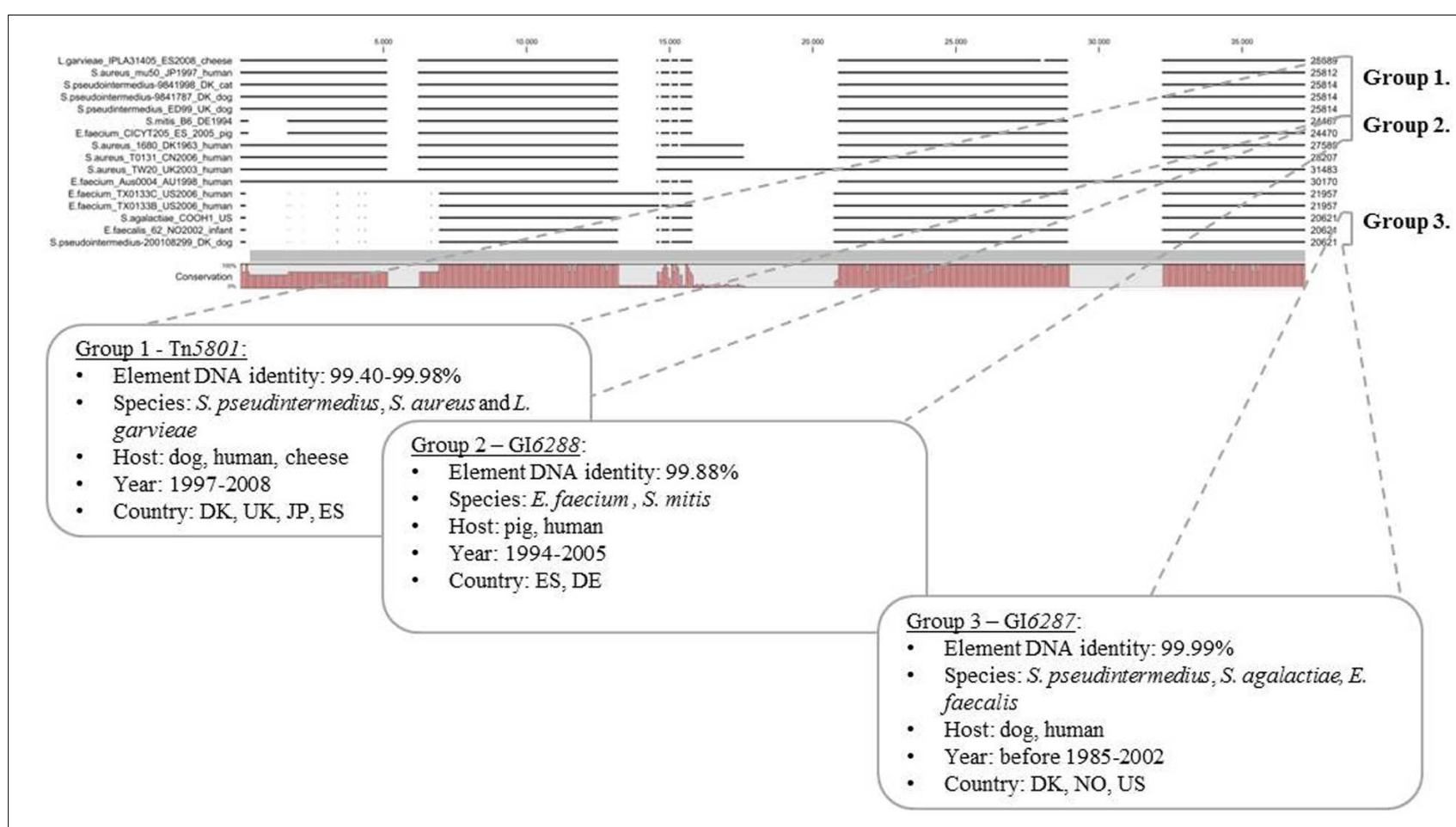

FIGURE 3 | Comparison of full length Tn5801-like Gls from this study (5) and full-length Tn5801-like elements detected in sequences from GenBank (11). A multiple alignment of selected Tn5801-like sequences constructed and visualized with CLC (version 7.5.1) revealed overall seven Tn5801 Gl types based on similar organization and DNA identity >99\%. Element sizes are shown at the end of the sequences (to the right). EFA, E. faecalis; SAG, Streptococcus agalactiae; SA, Staphylococcus aureus; SP: LG, Lactococcus garvieae; SM, Streptococcus mitis. (EF_TX0133C/Bahaman: two strains TX0133C and TX0133B).

to transfer Tn5801-like elements from S. pseudintermedius 200108299-1 or 9841998-1 to recipient strains, E. faecalis JH22, E. faecium BH4105, S. aureus $8794 \mathrm{RF}$ or S. aureus 4220RF, by filter-mating (data not shown). This was also the case in a study reporting a Tn5801-like element from $S$. agalactiae (Tn5801.Sag), for which circularization and conjugal transfer could not be shown (Mingoia et al., 2013). Apparently, horizontal transfer of Tn5801-like GIs occurs at extremely low frequencies and/or under highly specific conditions. These elements do not contain xis-like genes which are required for excision. Thus Tn5801-like elements are most likely mobilizable genomic islands (MGIs) that need a helper element providing them with a factor allowing their excision (Bellanger et al., 2014). Tn5801-like elements could also turn out to be true ICEs that encode their own excision, transfer by conjugation and their integration. However, we can not rule out that these elements may have spread in bacterial populations by transformation or transduction.

Overall, different types of Tn5801-like GIs were found to be present in publicly available sequences from Enterococcus, Lactobacillus-, Lactococcus-, Staphylococcus-, Streptococcus-, and Clostridium species associated with humans, animals or foods in Europe, USA, Asia, and Australia. This does show that successful dissemination of different types of this element has occurred among Gram-positive species world-wide. The observed diversity among the Tn5801-like GIs suggests plasticity of this element-type within Gram-positives, which is supported by the characterization of Tn6014 in S. aureus - Tn6014 contains a smaller region with a predicted integrase and a transposase gene, which may derive from Enterococcus.

Tn5801-like GIs can be dated back to the 1950's in human S. aureus (de Vries et al., 2009). In Enterococcus, a Tn5801-like GI is present in the human E. faecium E1636 strain isolated in 1961 (Supplementary Table S8; van Schaik et al., 2010). This is not many years after tetracycline resistance was first observed, and considering the complexity of these GIs it is highly unlikely that these elements should have evolved within only a few years. Thus, Tn5801-like (and Tn916-like) elements may have evolved long before tetracycline was introduced into the clinical practice and have transferred into human pathogenic species early in the so called "antibiotic era." Tn5801-like GIs could also have existed in human pathogens or reservoirs of pathogens prior to the "antibiotic era" and evolved by acquiring the tet $(\mathrm{M})$ gene.

\section{CONCLUSION}

This study presents evidence which supports that pet-associated S. pseudintermedius is a likely reservoir of Tn5801-like GIs detected in human pathogens (e.g., S. aureus). However, larger full-genome sequencing and metagenomic sequencing studies of animal-associated isolates/samples from an expanded time-period (including the pre-antibiotic era) should be 
conducted in order to make a final conclusion regarding the origin of Tn5801-like GIs in human pathogens such as S. aureus. Besides, the mechanism of transfer for Tn5801-like elements remains to be shown.

\section{AUTHOR CONTRIBUTIONS}

LV and YA designed the study and did most analysis as well as data interpretation of the work. $\mathrm{HH}$ contributed substantially to analysis and data interpretation regarding the genome sequencing. SR did experimental work and data interpretation regarding PCR-screenings. LV drafted the paper and all coauthors (YA, HA, and SR) have critically revised it.

\section{FUNDING}

The study was supported by Metropolitan University College, Department of Technology and by the Center for Genomic

\section{REFERENCES}

Aarestrup, F. M., Agerso, Y., Ahrens, P., Jorgensen, J. C., Madsen, M., and Jensen, L. B. (2000a). Antimicrobial susceptibility and presence of resistance genes in staphylococci from poultry. Vet. Microbiol. 74, 353-364. doi: 10.1016/S03781135(00)00197-8

Aarestrup, F. M., Agerso, Y., Gerner-Smidt, P., Madsen, M., and Jensen, L. B. (2000b). Comparison of antimicrobial resistance phenotypes and resistance genes in Enterococcus faecalis and Enterococcus faecium from humans in the community, broilers, and pigs in Denmark. Diagn. Microbiol. Infect. Dis. 37, 127-137. doi: 10.1016/S0732-8893(00) 00130-9

Agerso, Y., Jensen, L. B., Givskov, M., and Roberts, M. C. (2002). The identification of a tetracycline resistance gene tet(M), on a Tn916-like transposon, in the that encode their own excision, their transfer by conjugation and their integrationBacillus cereus group. FEMS Microbiol. Lett. 214, 251-256. doi: 10.1111/j.1574-6968.2002. tb11355.X

Agerso, Y., Pedersen, A. G., and Aarestrup, F. M. (2006). Identification of Tn5397like and Tn916-like transposons and diversity of the tetracycline resistance gene tet(M) in enterococci from humans, pigs and poultry. J. Antimicrob. Chemother. 57, 832-839. doi: 10.1093/jac/dkl069

Angiuoli, S. V., Gussman, A., Klimke, W., Cochrane, G., Field, D., Garrity, G., et al. (2008). Toward an online repository of standard operating procedures (SOPs) for (meta)genomic annotation. OMICS 12, 137-141. doi: 10.1089/omi.2008.0017

Bannoehr, J., and Guardabassi, L. (2012). Staphylococcus pseudintermedius in the dog: taxonomy, diagnostics, ecology, epidemiology and pathogenicity. Vet. Dermatol. 23, 1-16. doi: 10.1111/j.1365-3164.2012. 01046.x

Bellanger, X., Payot, S., Leblond-Bourget, N., and Guedon, G. (2014). Conjugative and mobilizable genomic islands in bacteria: evolution and diversity. FEMS Microbiol. Rev. 38, 720-760. doi: 10.1111/1574-6976. 12058

Ben Zakour, N. L., Beatson, S. A., van den Broek, A. H. M., Thoday, K. L., and Fitzgerald, J. R. (2012). Comparative genomics of the Staphylococcus intermedius group of animal pathogens. Front. Cell. Infect. Microbiol 2:44. doi: 10.3389/fcimb.2012.00044

Bennett, P. M. (2008). Plasmid encoded antibiotic resistance: acquisition and transfer of antibiotic resistance genes in bacteria. Br. J. Pharmacol. 153(Suppl.), S347-S357. doi: 10.1038/sj.bjp.0707607
Epidemiology ${ }^{10}$ grant 09-067103/DSF from the Danish Council for Strategic Research.

\section{ACKNOWLEDGMENTS}

Parts of this study were presented at the EMBO/EMBL Symposium: New Approaches and Concepts in Microbiology 2015. We want to thank Jacob D. Jensen, Hanne Mordhorst, Christina Aaby Svendsen, and Inge M. Hansen for excellent technical assistance and to Jan S. Knudsen for proofreading the manuscript.

\section{SUPPLEMENTARY MATERIAL}

The Supplementary Material for this article can be found online at: http://journal.frontiersin.org/article/10.3389/fmicb. 2016.00576

${ }^{10}$ Center for Genomic Epidemiology: http://www.genomicepidemiology.org/

Brede, D. A., Snipen, L. G., Ussery, D. W., Nederbragt, A. J., and Nes, I. F. (2011). Complete genome sequence of the commensal Enterococcus faecalis 62 , isolated from a healthy norwegian infant. J. Bacteriol. 193, 2377-2378. doi: 10.1128/JB.00183-11

Brenciani, A., Tiberi, E., Tili, E., Mingoia, M., Palmieri, C., Varaldo, P. E., et al. (2014). Genetic determinants and elements associated with antibiotic resistance in viridans group streptococci. J. Antimicrob. Chemother. 69, 1197-1204. doi: 10.1093/jac/dkt495

Burrus, V., Pavlovic, G., Decaris, B., and Guedon, G. (2002). Conjugative transposons: the tip of the iceberg. Mol. Microbiol. 46, 601-610. doi: 10.1046/j.1365-2958.2002.03191.x

Chopra, I., and Roberts, M. (2001). Tetracycline antibiotics: mode of action, applications, molecular biology, and epidemiology of bacterial resistance. Microbiol. Mol. Biol. Rev. 65, 232-260. doi: 10.1128/MMBR.65.2. 232-260.2001

Da Costa, P. M., Loureiro, L., and Matos, A. J. F. (2013). Transfer of multidrugresistant bacteria between intermingled ecological niches: the interface between humans, animals and the environment. Int. J. Environ. Res. Public Health 10, 278-294. doi: 10.3390/ijerph10010278

Da Cunha, V., Davies, M. R., Douarre, P.-E., Rosinski-Chupin, I., Margarit, I., Spinali, S., et al. (2014). Streptococcus agalactiae clones infecting humans were selected and fixed through the extensive use of tetracycline. Nat. Commun. 5:4544. doi: 10.1038/ncomms5544

Davies, J., and Davies, D. (2010). Origins and evolution of antibiotic resistance. Microbiol. Mol. Biol. Rev. 74, 417-433. doi: 10.1128/MMBR.00016-10

de Vries, L. E., Christensen, H., Skov, R. L., Aarestrup, F. M., and Agerso, Y. (2009). Diversity of the tetracycline resistance gene tet(M) and identification of Tn916- and Tn5801-like (Tn6014) transposons in Staphylococcus aureus from humans and animals. J. Antimicrob. Chemother. 64, 490-500. doi: 10.1093/jac/ dkp214

Denapaite, D., Bruckner, R., Nuhn, M., Reichmann, P., Henrich, B., Maurer, P., et al. (2010). The genome of Streptococcus mitis B6-what is a commensal? PLoS ONE 5:e9426. doi: 10.1371/journal.pone.0009426

Franke, A. E., and Clewell, D. B. (1981). Evidence for a chromosome-borne resistance transposon (Tn916) in Streptococcus faecalis that is capable of "conjugal" transfer in the absence of a conjugative plasmid. J. Bacteriol. 145, 494-502.

Guardabassi, L., Loeber, M. E., and Jacobson, A. (2004a). Transmission of multiple antimicrobial-resistant Staphylococcus intermedius between dogs affected by deep pyoderma and their owners. Vet. Microbiol. 98, 23-27. doi: 10.1016/j.vetmic.2003.09.021 
Guardabassi, L., Schwarz, S., and Lloyd, D. H. (2004b). Pet animals as reservoirs of antimicrobial-resistant bacteria. J. Antimicrob. Chemother. 54, 321-332. doi: 10.1093/jac/dkh332

Holden, M. T. G., Lindsay, J. A., Corton, C., Quail, M. A., Cockfield, J. D., Pathak, S., et al. (2010). Genome sequence of a recently emerged, highly transmissible, multi-antibiotic- and antiseptic-resistant variant of methicillinresistant Staphylococcus aureus, sequence type 239 (TW). J. Bacteriol. 192, 888-892. doi: 10.1128/JB.01255-09

Ito, T., Okuma, K., Ma, X. X., Yuzawa, H., and Hiramatsu, K. (2003). Insights on antibiotic resistance of Staphylococcus aureus from its whole genome: genomic island SCC. Drug Resist. 6, 41-52. doi: 10.1016/S1368-7646(03)00003-7

Jurado-Rabadán, S., de la Fuente, R., Ruiz-Santa-Quiteria, J. A., Orden, J. A., de Vries, L. E., and Agersø, Y. (2014). Detection and linkage to mobile genetic elements of tetracycline resistance gene tet(M) in Escherichia coli isolates from pigs. BMC Vet. Res. 10:155. doi: 10.1186/1746-6148-10-155

Kim, T. J., Na, Y. R., and Lee, J. I. (2005). Investigations into the basis of chloramphenicol and tetracycline resistance in Staphylococcus intermedius isolates from cases of pyoderma in dogs. J. Vet. Med. Ser. B Infect. Dis. Vet. Public Health 52, 119-124. doi: 10.1111/j.1439-0450.2005.00836.x

Kuroda, M., Ohta, T., Uchiyama, I., Baba, T., Yuzawa, H., Kobayashi, I., et al. (2001). Whole genome sequencing of methicillin-resistant Staphylococcus aureus. Lancet 357, 1225-1240. doi: 10.1016/S0140-6736(00) 04403-2

Larsen, M. V., Cosentino, S., Lukjancenko, O., Saputra, D., Rasmussen, S., Hasman, H., et al. (2014). Benchmarking of methods for genomic taxonomy. J. Clin. Microbiol. 52, 1529-1539. doi: 10.1128/JCM.02981-13

Larsen, M. V., Cosentino, S., Rasmussen, S., Friis, C., Hasman, H., Marvig, R. L., et al. (2012). Multilocus sequence typing of total-genome-sequenced bacteria. J. Clin. Microbiol. 50, 1355-1361. doi: 10.1128/JCM.06094-11

Li, H., Zhao, C., Chen, H., Zhang, F., He, W., Wang, X., et al. (2013). Identification of gene clusters associated with host adaptation and antibiotic resistance in chinese Staphylococcus aureus isolates by microarray-based comparative genomics. PLoS ONE 8:e53341. doi: 10.1371/journal.pone. 0053341

Marshall, B. M., and Levy, S. B. (2011). Food animals and antimicrobials: impacts on human health. Clin. Microbiol. Rev. 24, 718-733. doi: 10.1128/CMR.00002-11

Mingoia, M., Morici, E., Tili, E., Giovanetti, E., Montanari, M. P., and Varaldo, P. E. (2013). Characterization of Tn5801.Sag, a variant of Staphylococcus aureus Tn916 family transposon Tn5801 that is widespread in clinical isolates of Streptococcus agalactiae. Antimicrob. Agents Chemother. 57, 4570-4574. doi: 10.1128/AAC.00521-13

Mullany, P., Roberts, A. P., and Wang, H. (2002). Mechanism of integration and excision in conjugative transposons. Cell Mol. Life Sci. 59, 2017-2022. doi: $10.1007 / \mathrm{s} 000180200001$

Rice, L. B. (1998). Tn916 family conjugative transposons and dissemination of antimicrobial resistance determinants. Antimicrob. Agents Chemother. 42, 1871-1877.

Roberts, A. P., Chandler, M., Courvalin, P., Guedon, G., Mullany, P., Pembroke, T., et al. (2008). Revised nomenclature for transposable genetic elements. Plasmid 60, 167-173. doi: 10.1016/j.plasmid.2008.08.001
Roberts, A. P., Johanesen, P. A., Lyras, D., Mullany, P., and Rood, J. I. (2001). Comparison of Tn5397 from Clostridium difficile, Tn916 from Enterococcus faecalis and the CW459tet(M) element from Clostridium perfringens shows that they have similar conjugation regions but different insertion and excision modules. Microbiology 147, 1243-1251. doi: 10.1099/00221287-147-5-1243

Roberts, A. P., and Mullany, P. (2009). A modular master on the move: the Tn916 family of mobile genetic elements. Trends Microbiol. 17, 251-258. doi: 10.1016/j.tim.2009.03.002

Roberts, A. P., and Mullany, P. (2011). Tn916-like genetic elements: a diverse group of modular mobile elements conferring antibiotic resistance. FEMS Microbiol. Rev. 35, 856-871. doi: 10.1111/j.1574-6976.2011.00283.x

Schwarz, S., Roberts, M. C., Werckenthin, C., Pang, Y., and Lange, C. (1998). Tetracycline resistance in Staphylococcus spp. from domestic animals. Vet. Microbiol. 63, 217-227. doi: 10.1016/S0378-1135(98)00234-X

Toussaint, A., and Merlin, C. (2002). Mobile elements as a combination of functional modules. Plasmid 47, 26-35. doi: 10.1006/plas.2001.1552

U.S. Department of Health and Human Services - Centers for Disease Control and Prevention [CDC] (2013). Antibiotic Resistance Threats in the United States, 2013. Available at: http://www.cdc.gov/drugresistance/threat-repor-2013/pdf/ af-threats-2013-508.pdf

van Schaik, W., Top, J., Riley, D. R., Boekhorst, J., Vrijenhoek, J. E. P., Schapendonk, C. M. E., et al. (2010). Pyrosequencing-based comparative genome analysis of the nosocomial pathogen Enterococcus faecium and identification of a large transferable pathogenicity island. BMC Genomics 11:239. doi: 10.1186/1471-2164-11-239

Wegener, H. C. (2012). “Antibiotic resistance - linking human and animal health," in Improving Food Safety Through A One Health Approach: Workshop Summary, ed. Institute of Medicine (US) (Washington, DC: National Academies Press (US)).

Wernli, D., Haustein, T., Conly, J., Carmeli, Y., Kickbusch, I., and Harbarth, S. (2011). A call for action: the application of the international health regulations to the global threat of antimicrobial resistance. PLoS Med. 8:e1001022. doi: 10.1371/journal.pmed.1001022

Wozniak, R. A., and Waldor, M. K. (2010). Integrative and conjugative elements: mosaic mobile genetic elements enabling dynamic lateral gene flow. Nat. Rev. Microbiol. 8, 552-563. doi: 10.1038/nrmicro2382

Zankari, E., Hasman, H., Cosentino, S., Vestergaard, M., Rasmussen, S., Lund, O., et al. (2012). Identification of acquired antimicrobial resistance genes. J. Antimicrob. Chemother. 67, 2640-2644. doi: 10.1093/jac/dks261

Conflict of Interest Statement: The authors declare that the research was conducted in the absence of any commercial or financial relationships that could be construed as a potential conflict of interest.

Copyright (C) 2016 de Vries, Hasman, Jurado Rabadán and Agersø. This is an openaccess article distributed under the terms of the Creative Commons Attribution License (CC BY). The use, distribution or reproduction in other forums is permitted, provided the original author(s) or licensor are credited and that the original publication in this journal is cited, in accordance with accepted academic practice. No use, distribution or reproduction is permitted which does not comply with these terms. 